\title{
The influence of bone mineral density and cortical index on the complexity of fractures of the proximal humerus
}

\section{J.W.A.M. den Teuling, \\ B.S. Pauwels, L. Janssen, C.E. Wyers, H. M. J. Janzing, J.P.W. van den Bergh, J. W. Morrenhof}

VieCuri Medical Centre, Venlo, The Netherlands
- J.W.A.M. den Teuling, MD, Orthopaedic Surgeon,

B.S. Pauwels, MD, Orthopaedic Resident,

- L. Janssen, PhD, Postdoctoral researcher,

H. M. J. Janzing, MD, PhD,

Trauma Surgeon,

J. W. Morrenhof, MD

$\mathrm{PhD}$, Orthopaedic Surgeon,

Department of Orthopaedic

Surgery, VieCuri Medical Centre, Venlo, The Netherlands.

- C.E. Wyers, PhD, Postdoctoral

researcher, Department of Internal Medicine,

- J.P.W. van den Bergh, Professor, MD, PhD, Internist /

Endocrinologist, Department of

Internal Medicine, VieCuri Medical

Centre and NUTRIM School

of Nutrition and Translational

Research in Metabolism,

Maastricht University Medical

Centre, Venlo and Maastricht, The

Netherlands.

Correspondence should be sent to J. den Teuling;

email: joost_den_teuling@

hotmail.com

doi: 10.1302/2046-3758.610 BJR-2017-0080

Bone Joint Res 2017;6:584-589.

\section{Objectives}

The goal of this study is to investigate the relation between indicators of osteoporosis (i.e., bone mineral density (BMD), and Cortical Index (CI)) and the complexity of a fracture of the proximal humerus as a result of a low-energy trauma.

\section{Methods}

A retrospective chart review of 168 patients (mean age 67.2 years, range 51 to 88.7) with a fracture of the proximal humerus between 2007 and 2011, whose BMD was assessed at the Fracture Liaison Service with Dual Energy X-ray Absorptiometry (DXA) measurements of the hip, femoral neck (FN) and/or lumbar spine (LS), and whose $\mathrm{Cl}$ and complexity of fracture were assessed on plain anteroposterior radiographs of the proximal humerus.

\section{Results}

No significant differences were found between simple and complex fractures of the proximal humerus in the BMD of the hip, FN or LS (all $p>0.3$ ) or in the $\mathrm{Cl}(p=0.14)$. Only the body mass index was significantly higher in patients with a complex fracture compared with those with a simple fracture $(26.9$ vs $25.2 ; p=0.05)$.

\section{Conclusion}

There was no difference in BMD of the hip, $\mathrm{FN}$, LS or $\mathrm{Cl}$ of the proximal humerus in simple compared with complex fractures of the proximal humerus after a low-energy trauma. Factors other than the BMD and $\mathrm{Cl}$, for example body mass index, may play a more important role in the complexity of this fracture.

Cite this article: Bone Joint Res 2017;6:584-589.

Keywords: Osteoporosis, Bone mineral density, Cortical Index, Proximal humerus, Fracture

\section{Article focus}

- To investigate the relationship between indicators of osteoporosis and the complexity of fractures of the proximal humerus.

\section{Key messages}

- Bone mineral density (BMD) of the hip, femoral neck, lumbar spine and the Cortical Index $(\mathrm{Cl})$ of the proximal humerus were not different in simple compared to complex fractures of the proximal humerus.

- Other factors, such as body mass index (BMI), might be more important in the complexity of fractures of the proximal humerus.

\section{Strengths and limitations}

- Large study population in which multiple indicators of osteoporosis (BMD in various regions and $\mathrm{Cl}$ ) were measured and the fracture was classified.

- Retrospective study design.

- Poor intra- and interobserver variability of the AO/OTA classification.

\section{Introduction}

Osteoporosis is a major cause of low-energy fractures in elderly patients. There has been an exponential growth in the prevalence of fractures of the proximal humerus after the fifth decade ${ }^{1}$ and they compromise about $4 \%$ of all fractures. ${ }^{1,2}$ The incidence is approximately 70 per 100000 and will probably 


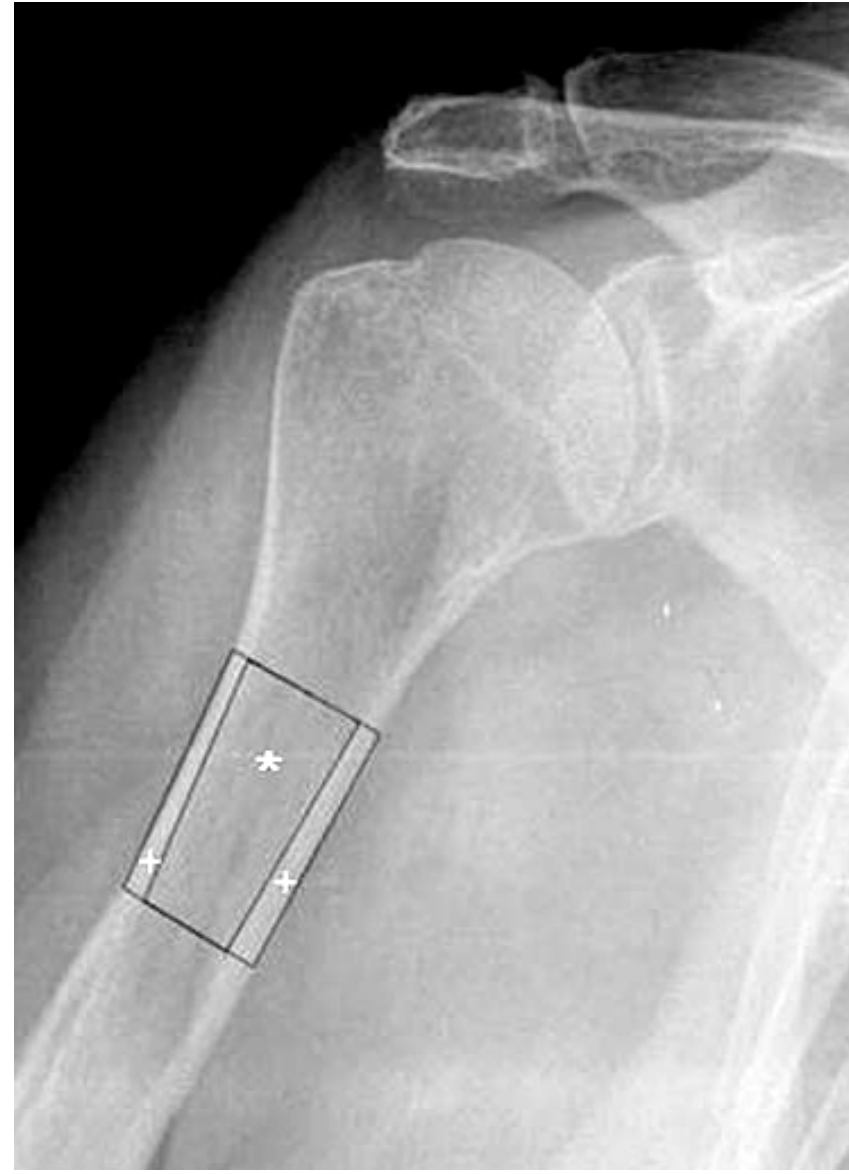

Fig. 1

Example for region of interest (ROI) for analysis of cortical index. * denotes the area of marrow cavity (MA). + denotes the area of the cortex. The area of the shaft $(T A)=$ the sum of * and both + . The cortical index $(C I)$ is calculated by using the following formula: $\mathrm{Cl}=(T \mathrm{~A}-\mathrm{MA}) / \mathrm{TA}$ or $\left[\left(^{*}\right.\right.$ and + medial and + lateral) $\left.-\left(^{*}\right)\right] /\left({ }^{*}\right.$ and + medial and + lateral).

increase due to the association of age and osteoporosis. In women aged 80 years and older, it is the third most common type of fracture, following that of the proximal femur and distal radius. ${ }^{3,4}$

The relationship between low bone mineral density (BMD) and fracture risk is well established. It is likely that bone density also plays a role in the complexity of fractures. A clear correlation between BMD and the severity of distal radial fractures has already been described. ${ }^{3}$

The current benchmark for diagnosing osteoporosis, according to the World Health Organization (WHO), is by BMD measurements with a dual energy $x$-ray absorptiometry (DXA) scan. ${ }^{5}$ Diederichs et $\mathrm{al}^{6}$ and Wilsonet $\mathrm{al}^{7}$ have reported differences in BMD for each anatomical area and therefore hip or lumbar spine BMD (as commonly measured) might not correspond to the BMD in the proximal humerus. Previous studies have proved that measurements of the cortical thickness in conventional radiographs are a reliable way of assessing osteoporosis. 8,9 So far, only one study has investigated the association between cortical thickness and fracture complexity of the proximal humerus. Osterhoff et al ${ }^{10}$ demonstrated that the cortical index $(\mathrm{Cl})$ (a measure of cortical thickness) was not significantly different between two-part fractures of the proximal humerus and complex fractures. However, these results were derived from a small population and larger studies are necessary to confirm these findings.

Until now, the link between BMD and the severity of a fracture of the proximal humerus has not been extensively investigated. The aim of this study was to analyse the relationship of BMD and cortical thickness, to the occurrence of complex fractures of the proximal humerus as a result of low-energy trauma. We hypothesised that osteoporosis (low BMD or cortical thickness) results in more complex fractures of the proximal humerus.

\section{Patients and Methods}

We performed a retrospective chart review of 175 consecutive patients who visited the Fracture Liaison Service (FLS) for fracture risk evaluation between 2007 and 2011. In our institution, patients aged 50 years and older with a proximal humeral fracture, as a result of low-energy trauma, were offered a systematic fracture risk evaluation at the FLS. ${ }^{11}$ The impact of the trauma was reported by the patients during the visit to the FLS and was documented for this study. Gender, age and body mass index (BMI) were also documented. In some patients, the T-scores for the hip $(n=6)$, femoral neck (FN; $n=7$ ) or lumbar spine ( $L N ; n=9)$ were missing. These patients were only excluded for the analyses which required the particular missing T-score.

Bone mineral density and cortical index. BMD was measured at the hip, FN and LS with DXA (Hologic, Inc., Marlborough, Massachusetts). For each of these regions, T-scores were obtained, representing normal BMD (T-scores $\geqslant-1)$, osteopaenia (T-scores -1 to -2.5 ) and osteoporosis (T-scores $\leqslant-2.5$ ), according to the WHO criteria. ${ }^{12}$ When distinguishing between normal BMD, osteopaenia or osteoporosis, the lowest T-score was the decisive factor. All patients had at least one evaluable DXA measurement of the three anatomical regions (hip, FN or LS).

The $\mathrm{Cl}$ indicates the relative thickness of the cortex in relation to the total shaft diameter. The $\mathrm{Cl}$ was measured using a method adapted from a technique described in previous studies (Fig. 1). ${ }^{10}$ Plain anteroposterior (AP) radiographs of the proximal humerus were used to obtain the $\mathrm{Cl}$. The best-quality radiograph taken within four weeks of the trauma was used. A digital radiographic image processing software with a digital measuring device (IMPAX 6.6.1; Agfa HealthCare N.V, Mortsel, Belgium) was used for measuring the $\mathrm{Cl}$. The interobserver variability for measuring the $\mathrm{Cl}$ and cortical thickness described by Tingart et al, ${ }^{9}$ was good, hence the analysis of the $\mathrm{Cl}$ was performed by a single investigator (BP). 


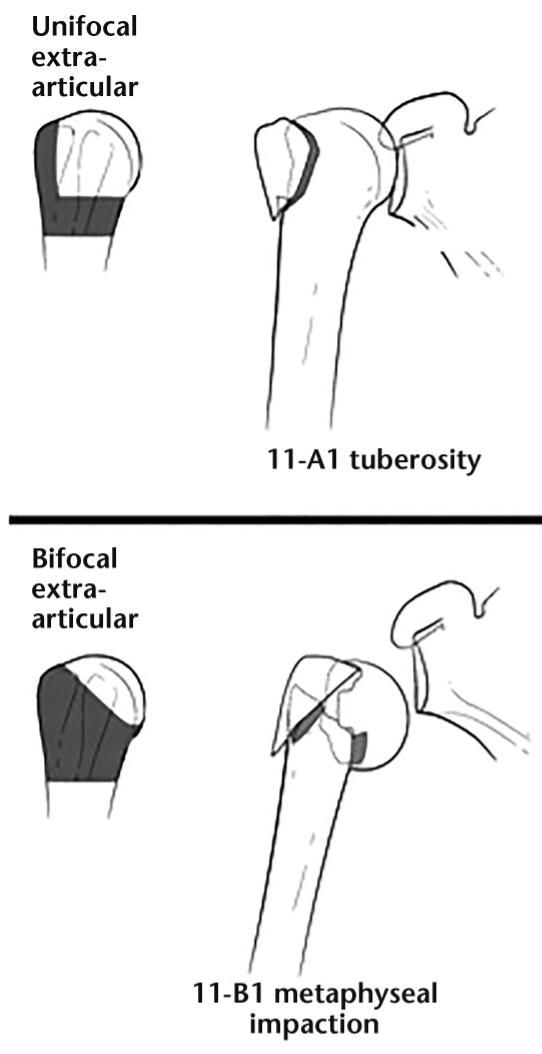

Articular
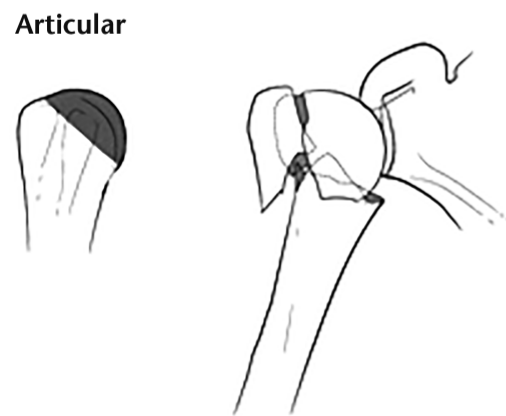

11-C1 with slight displacement

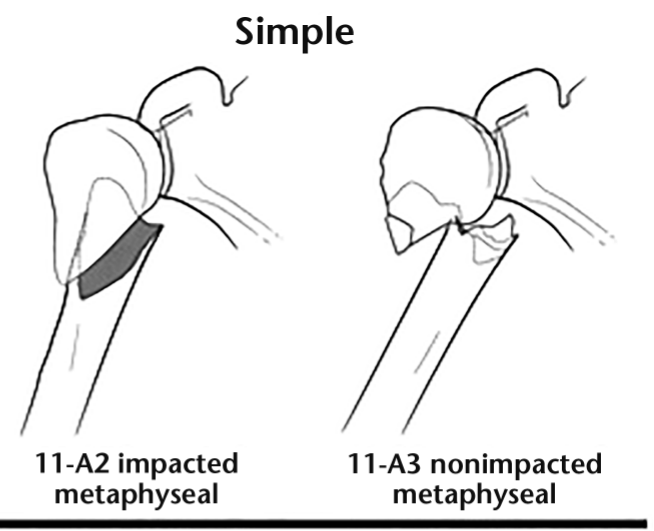

Complex

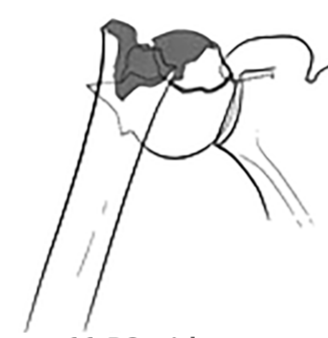

11-B2 without metaphyseal impaction

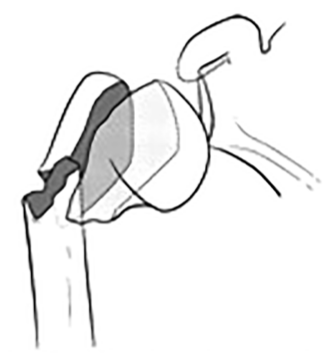

11-B3 with glenohumeral dislocation

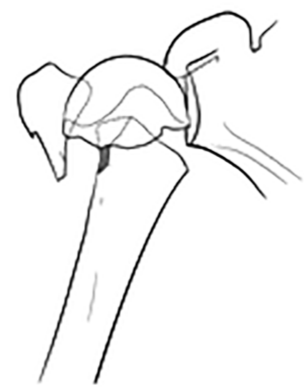

11-B2 impacted with marked displacement

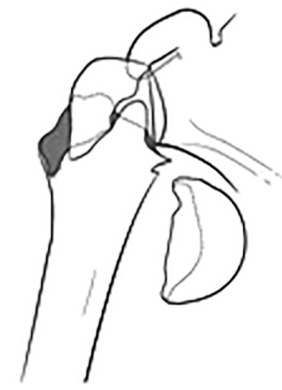

11-B3 disclocated

Fig. 2

Proximal humeral fractures divided into simple and complex fractures using the AO/OTA criteria.

Complexity of fracture. Plain AP radiographs of the proximal humerus (the same as used for obtaining the $\mathrm{Cl}$ ) were classified according to the AO/OTA classification. ${ }^{13}$ Next, fractures were divided into "type A" or "simple" fractures, and "type $B / C$ " or "complex" fractures (Fig.2). This assessment was independently carried out by two investigators (JT and BP). In the event of a disagreement between the two investigators, a third independent senior investigator (JM) decided on the definitive classification.

Statistical analysis. Data analysis was performed using SPSS statistics software version 21.0 (IBM Corp, Armonk, NY). Patients were characterised by age, gender, mean
T-scores, BMD (normal/osteopaenia/osteoporosis), mean $\mathrm{Cl}$ and type of fracture (simple/complex). Continuous outcome variables were checked for a normal distribution. To study the differences in mean T-scores and $\mathrm{Cl}$ between the simple and complex fracture groups, independent samples $t$-tests were used. This relationship was also studied by testing whether the frequencies of osteoporosis and osteopaenia were equally distributed among simple and complex fracture groups, using a chi-squared test. In addition, we tested whether there was a correlation between T-scores and $\mathrm{Cl}$ using Pearson's correlation coefficients. 
Table I. Differences in mean age, gender, mean T-scores, osteoporosis classification and mean cortical index between simple and complex fractures of the proximal humerus

\begin{tabular}{|c|c|c|c|c|}
\hline Descriptives & All $(n=168)$ & Simple fracture $(n=46)$ & Complex fracture $(n=122)$ & p-value \\
\hline Mean age, yrs (SD) & $67.2(9.4)$ & $68.5(9.7)$ & $66.8(9.3)$ & 0.29 \\
\hline \multicolumn{5}{|l|}{ Gender, n (\%) } \\
\hline Male & $24(14)$ & $5(11)$ & $19(16)$ & 0.44 \\
\hline Female & $144(86)$ & $41(89)$ & $103(84)$ & 0.44 \\
\hline Mean body mass index (SD) & $26.9(4.7)$ & $25.2(4.1)$ & $26.9(4.7)$ & 0.05 \\
\hline \multicolumn{5}{|l|}{ Mean BMD in T-scores* (SD) } \\
\hline Hip & $-1.42(0.95)$ & $-1.53(0.99)$ & $-1.38(0.93)$ & 0.38 \\
\hline $\mathrm{FN}$ & $-1.81(0.94)$ & $-1.90(0.92)$ & $-1.78(0.95)$ & 0.47 \\
\hline LS & $-1.82(1.34)$ & $-2.00(1.22)$ & $-1.76(1.37)$ & 0.31 \\
\hline \multicolumn{5}{|c|}{ Osteoporosis classification, n (\%) } \\
\hline Normal & $23(14)$ & $6(13)$ & $17(14)$ & 0.50 \\
\hline Osteopaenia & $73(43)$ & $17(37)$ & $56(46)$ & 0.50 \\
\hline Osteoporosis & $72(43)$ & $23(50)$ & $49(40)$ & 0.50 \\
\hline Mean cortical index (SD) & $0.231(0.053)$ & $0.221(0.053)$ & $0.235(0.053)$ & 0.14 \\
\hline
\end{tabular}

*Not all T-scores are collected: Complex fracture group T-score Hip $\mathrm{n}=116, \mathrm{~T}$-score Femoral Neck $\mathrm{n}=115, \mathrm{~T}$-score Lumbar Spine $\mathrm{n}=116$. In the simple fracture group, T-score Lumbar Spine $n=43$

$p$-values calculated using independent samples $t$-tests

BMD, bone mineral density; FN, femoral neck; LS, lumbar spine

Consequently, we studied the effect of gender, age and BMI on our primary outcome measures. The independent samples $t$-test was used to investigate differences in age between the simple and complex fracture group, and to examine differences in $\mathrm{T}$-scores and $\mathrm{Cl}$ between men and women.

Finally, we investigated the relationship between T-scores and fracture complexity, and between $\mathrm{Cl}$ and fracture complexity, adjusted for age, gender and BMI, using a logistic regression analysis.

\section{Results}

Patient characteristics. In this retrospective chart review study, 175 patients with a proximal humeral fracture visited the FLS. Seven patients were excluded from this study because of unusual AP radiographs of the proximal humerus $(n=2)$, a previous humeral fracture $(n=1)$, incomplete fracture lines or disruption at the location of the $\mathrm{Cl}$ measurements $(\mathrm{n}=3)$, and a high-energy trauma and additional injuries to the humerus $(\mathrm{n}=1)$. A total of 168 patients ( 24 men and 144 women) with a mean age of 67.2 years (SD 9.4, range 51.0 to 88.7 ) were included for analysis. All patient characteristics are listed in Table I. The humeral fractures in all included patients resulted from low-energy trauma. A direct fall on the shoulder from standing height was the most frequent trauma mechanism. According to the AO/OTA classification, 46 fractures were classified as "simple" and 122 fractures were classified as "complex". The median time between the radiograph used for the measurements $(\mathrm{Cl}$ and fracture classification) and the FLS visit was 68 days (interquartile range 39 to 101 days).

BMD and $\mathrm{CI}$ in simple and complex fractures. Based on the T-scores, 23 (14\%) patients had a normal BMD, 73 (43\%) patients had osteopaenia, and 72 (43\%) osteoporosis. No significant differences were found between simple and complex fractures of the proximal humerus in the mean T-score of the hip (respectively, 1.53 and -1.38 , $p=0.38$ ), FN (respectively, -1.90 and $-1.78, p=0.47$ ) or LS (respectively, -2.00 and $-1.76, p=0.31$ ). Accordingly, the proportions of simple and complex fractures were not significantly different between patients with normal $\mathrm{BMD}$, osteopaenia or osteoporosis. In addition, the mean $\mathrm{Cl}$ of the simple and complex fracture groups (0.221 versus $0.235, p=0.14$ ) were also not significantly different (Table I).

The $\mathrm{Cl}$ showed a moderate correlation with T-scores of the hip ( $r=0.38, p<0.01)$ and the FN $(r=0.41$, $p<0.01$ ), and only a weak correlation with the T-score of the $L S(r=0.18, p=0.03)$.

Gender, age and BMI. The mean age and the gender distribution of the patients in the simple fracture group was similar to that of the patients in the complex fracture group (age: 68.5 versus 66.8 respectively, $p=0.29$; male gender: $11 \%$ versus $16 \%$ respectively, $p=0.44$ ). Mean T-scores and cortical index were not significantly different between men and women (Table II). The mean $\mathrm{BMI}$ in the complex fracture group was significantly higher than that in the simple fracture group (26.9 versus 25.2; $p=0.05)$. When adjusted for age, gender and the BMD using logistic regression analysis, the effect of BMI on fracture complexity remained marginally significant, depending on the T-scores used to determine the BMD (odds ratio $=1.09$ to $1.10 ; p=0.05$ to 0.08 ).

Finally, age, gender and BMI did not qualitatively influence the relationship between the BMD and fracture complexity ( $p>0.6$ for all T-scores), or between the $\mathrm{Cl}$ and fracture complexity $(p=0.23)$.

\section{Discussion}

Study goal. This is the largest study to date looking at $\mathrm{BMD}$ and the $\mathrm{Cl}$ of the proximal humerus, in relation to the occurrence of complex fractures of the proximal humerus. In contrast to the authors' hypothesis, 
Table II. Mean differences in T-scores and cortical indexbetween men and women

\begin{tabular}{lrll}
\hline & n & Mean (sD) & p-value \\
\hline T-score hip & 22 & $-1.43(0.79)$ & 0.98 \\
Men & 140 & $-1.42(0.97)$ & 0.98 \\
Women & & & \\
T-score FN & 21 & $-1.85(0.86)$ & 0.84 \\
Men & 140 & $-1.80(0.96)$ & 0.84 \\
Women & & & \\
T-score LS & 23 & $-1.60(1.64)$ & 0.38 \\
Men & 136 & $-1.86(1.28)$ & 0.38 \\
Women & & & \\
Cortical index & 24 & $0.237(0.042)$ & 0.58 \\
Men & 144 & $0.230(0.054)$ & 0.58 \\
Women & &
\end{tabular}

p-values calculated using independent samples $t$-tests

FN, femoral neck; LS, lumbar spine

osteoporosis does not result in more complex fractures of the proximal humerus. However, the BMI was significantly higher in patients with complex fractures.

Relation between osteoporosis and fracture complexity. The current benchmark for diagnosing osteoporosis or osteopaenia is the DXA scan. Despite DXA being regarded as the best possible option for assessing the BMD in different anatomical sites, $6,7,14$ this diagnostic tool is not without its failings. Richmond et al $^{15}$ have showed that the accuracy of this test depends on the placement and sizing of the region of interest (ROI), overlying material in the ROI (fat or soft-tissue calcifications) and the absence of normal structures (e.g. after laminectomy). As cadaveric studies show, osteoporosis plays an important role in the failure of osteosynthesis in fracture treatment. ${ }^{16-18}$ The quality of bone post-trauma may play a more important role in the future to optimise treatment of fractures of the proximal humerus. ${ }^{19}$ In contrast to a previous study in which a clear correlation was found between the BMD (of the hip) and the severity of distal radial fractures, we did not find the same association between the BMD (of the hip, FN or LS) and fractures of the proximal humerus. ${ }^{3}$

The absolute cortical thickness and the $\mathrm{Cl}$ have proven to be reliable ways of assessing osteoporosis from conventional radiographs in previous studies. 8,9 The advantage of the $\mathrm{Cl}$ over the cortical thickness is that the $\mathrm{Cl}$ does not depend on magnification differences between images. In contrast to the DXA scan, the $\mathrm{Cl}$ can easily be measured directly after trauma and it is determined using conventional radiographs. Therefore, it is a measurement for assessing bone quality and can be used to select the best treatment option following a fracture of the proximal humerus. Osterhoff et al $^{10}$ demonstrated in a small population that the $\mathrm{Cl}$ was not significantly different between two-part fractures of the proximal humerus and complex fractures. In the present study, with a larger cohort of patients, we found a similar result and therefore can confirm their findings.
There are weak to moderate correlation between the T-scores (hip, FN and LS) and the $\mathrm{Cl}$ of the proximal humerus. As other studies showed, the BMD varies for each anatomical area and therefore the hip, FN or LS BMD (as commonly measured) might not correspond to the BMD of the proximal humerus. ${ }^{6,7}$ Tingart et al, ${ }^{9}$ however, found significant good to excellent correlations between T-scores of different sites within the proximal humerus and the absolute cortical thickness of the proximal humerus ( $r=0.64$ to 0.84 ). However, in daily practice, only the BMD of the hip, FN and LS are commonly measured to evaluate a patient's bone quality. Although these parameters might give an idea about the osteoporotic status in general, they seem inadequate to give detailed information about the proximal humerus in particular. The $\mathrm{Cl}$ appears to be an appropriate alternative for evaluating local bone quality.

Effects of gender, age and BMI. In contrast to a previous study that showed that older women experienced a greater decline in BMD than men of the same age, ${ }^{20}$ our results do not show an association between BMD or $\mathrm{Cl}$ and gender. As for other studies, our results show a decrease in $\mathrm{BMD}$ and $\mathrm{Cl}$ in relation to increasing age. ${ }^{21-23}$ Nevertheless, age does not seem to be a discriminating factor for a simple or complex proximal humeral fracture.

In this study, we found a significantly higher BMl in the complex fracture group than in the simple fracture group. Previous studies have already shown that populations with a higher BMI are more at risk of fracturing a proximal humerus than people with normal or low BMI. ${ }^{24,25}$ In a large population-based study, Prieto-Alhambra et al24 found that post-menopausal women with obesity are protected against hip and pelvic fractures but have an almost $30 \%$ increased risk of fracturing the proximal humerus in comparison with normal/underweight women. They indicated that the reason might be related to the trauma mechanism and the padding provided by adipose tissue. It is suggested that people with higher body weight experience an increased traumatic force during a fall. According to our data, a higher BMI results in increased BMD of the femur (T-scores of the hip and FN). Beck et al ${ }^{26}$ suggest that as the hip is a weight bearing joint, the greater force of soft-tissue mass in heavier individuals stimulates the bone to increase the BMD in this region.

Study limitations. The retrospective design is the most important limitation in this study. Due to this design, we could not investigate the influence of other variables on the complexity of fractures of the proximal humerus, such as the specific trauma mechanism. Osterhoff et al ${ }^{10}$ also studied the relationship between the $\mathrm{Cl}$ and the complexity of fractures of the proximal humerus after low-energy trauma. They observed that more falls from a certain height were seen in the complex fracture group than in the simple fracture group. This might suggest that trauma mechanism plays a more important role in the complexity of fractures of the proximal humerus than 
bone parameters such as $\mathrm{BMD}$ or $\mathrm{Cl}$, although this has not yet been investigated. Another limitation is the minimal intra- and interobserver variability of the AO/OTA classification. ${ }^{27,28}$ Nevertheless, in our opinion it is the most suitable method for classifying simple and complex fractures of the proximal humerus. To optimise reliability, the classification was independently carried out by two investigators (JT) and (BP) and, in the event of disagreement, a third independent investigator (JM) would decide on the definitive classification. Finally, the DXA scans were not performed immediately after the trauma, which could have resulted in changes to the bone parameters over time. However, the median time between the radiographs performed, used for the $(\mathrm{Cl}$ and fracture classification) measurements and the DXA was only two months. This period was similar for both the simple and complex fracture groups. Therefore, we deemed the influence on our results of possible bone changes in this period to be minimal.

In conclusion, this study shows no difference in the $\mathrm{BMD}$ of the hip, $\mathrm{FN}$ or $\mathrm{LS}$, and no difference in the $\mathrm{Cl}$ of the proximal humerus, in simple compared with complex fractures of the proximal humerus after a low-energy trauma. However, BMI in the complex fracture group is significantly higher than in the simple fracture group.

Consequently, a simple proximal humeral fracture does not necessarily imply a better bone quality than would be found in a complex fracture. These results suggest that factors other than the BMD and the $\mathrm{Cl}$, such as the BMI, play a more important role in the complexity of fractures of the proximal humerus. Further studies should focus on the relationship between the BMD of the proximal humerus and the complexity of a fracture of the proximal humerus, and also the role of trauma mechanisms on the complexity of these fractures.

\section{References}

1. Kristiansen B, Barfod G, Bredesen J, et al. Epidemiology of proximal humeral fractures. Acta Orthop Scand 1987;58:75-77.

2. Court-Brown CM, Caesar B. Epidemiology of adult fractures: A review. Injury 2006;37:691-697.

3. Clayton RAE, Gaston MS, Ralston SH, Court-Brown CM, McQueen MM. Association between decreased bone mineral density and severity of distal radial fractures. J Bone Joint Surg [Am] 2009;91-A:613-619.

4. Court-Brown CM, Garg A, McQueen MM. The epidemiology of proximal humeral fractures. Acta Orthop Scand 2001;72:365-371.

5. Kanis JAon behalf of the World Health Organization Scientific Group. Assessment of osteoporosis at the primary health-care level. https://www .sheffield.ac.uk/FRAX/pdfs/WHO_Technical_Report.pdf (date last accessed 22 September 2017).

6. Diederichs G, Korner J, Goldhahn J, Linke B. Assessment of bone quality in the proximal humerus by measurement of the contralateral site: a cadaveric analyze. Arch Orthop Trauma Surg 2006;126:93-100.

7. Wilson J, Bonner TJ, Head M, et al. Variation in bone mineral density by anatomical site in patients with proximal humeral fractures. J Bone Joint Surg $[\mathrm{Br}]$ 2009;91-B:772-775.

8. Barnett E, Nordin BEC. The radiological diagnosis of osteoporosis: a new approach. ClinRadiol 1960;11:166-174.

9. Tingart MJ, Apreleva M, von Stechow D, Zurakowski D, Warner JJ. The cortical thickness of the proximal humeral diaphysis predicts bone mineral density of the proximal humerus. J Bone Joint Surg [Br]2003;85-B:611-617.
10. Osterhoff G, Diederichs G, Tami A, et al. Influence of trabecular microstructure and cortical index on the complexity of proximal humeral fractures. Arch Orthop Trauma Surg 2012;132:509-515.

11. Bours SP, van Geel TA, Geusens PP, et al. Contributors to secondary osteoporosis and metabolic bone diseases in patients presenting with a clinical fracture. J Clin Endocrinol Metab 2011;96:1360-1367.

12. Kanis JA, Melton LJ III, Christiansen C, Johnston CC, Khaltaev N. The diagnosis of osteoporosis. J Bone Miner Res 1994;9:1137-1141.

13. Marsh JL, Slongo TF, Agel J, et al. Fracture and dislocation classification compendium - 2007: orthopaedic Trauma Association classification, database and outcomes committee. J Orthop Trauma 2007;21(Suppl):S1-S133.

14. Doetsch AM, Faber J, Lynnerup N, et al. Bone mineral density measurement over the shoulder region. Calcif Tissue Int 2002;71:308-314.

15. Richmond B. DXA scanning to diagnose osteoporosis: do you know what the results mean? Cleve Clin J Med 2003;70:353-360.

16. Tingart MJ, Lehtinen J, Zurakowski D, Warner JJP, Apreleva M. Proximal humeral fractures: regional differences in bone mineral density of the humeral head affect the fixation strength of cancellous screws. J Shoulder Elbow Surg 2006; 15:620-624

17. Fankhauser F, Schippinger G, Weber K, et al. Cadaveric-biomechanical evaluation of bone-implant construct of proximal humerus fractures (Neer type 3). J Trauma 2003:55:345-349.

18. Sjöstedt A, Zetterberg C, Hansson T, Hult E, Ekström L. Bone mineral content and fixation strength of femoral neck fractures. A cadaver study. Acta Orthop Scand 1994;65:161-165.

19. Krappinger D, Bizzotto N, Riedmann S, et al. Predicting failure after surgical fixation of proximal humerus fractures. Injury 2011;42:1283-1288.

20. Hannan MT, Felson DT, Anderson JJ. Bone mineral density in elderly men and women: results from the Framingham osteoporosis study. J Bone Miner Res 1992;7:547-553.

21. Sambrook P, Cooper C. Osteoporosis. Lancet 2006;367:2010-2018

22. Johnell 0, Kanis J. Epidemiology of osteoporotic fractures. Osteoporos Int 2005;16(Suppl 2):S3-S7.

23. MantilaRoosa SM, Hurd AL, Xu H, Fuchs RK, Warden SJ. Age-related changes in proximal humerus bone health in healthy, white males. Osteoporos Int 2012;23: 2775-2783.

24. Prieto-Alhambra D, Premaor MO, FinaAvilés F, et al. The association between fracture and obesity is site-dependent: a population-based study in postmenopausal women.J Bone Miner Res 2012;27:294-300.

25. Gnudi S, Sitta E, Lisi L. Relationship of body mass index with main limb fragility fractures in postmenopausal women. J Bone Miner Metab 2009;27:479-484.

26. Beck TJ, Petit MA, Wu G, et al.Does obesity really make the femur stronger? $B M D$, geometry, and fracture incidence in the women's health initiativeobservational study. J Bone Miner Res 2009;24:1369-1379.

27. Foroohar A, Tosti R, Richmond JM, Gaughan JP, Ilyas AM. Classification and treatment of proximal humerus fractures: inter-observer reliability and agreement across imaging modalities and experience. J Orthop Surg Res 2011;6:38

28. Bruinsma WE, Guitton TG, Warner JJ, Ring DD, Science of Variation Group. Interobserver reliability of classification and characterization of proximal humeral fractures: a comparison of two and three-dimensional CT. J Bone Joint Surg [Am]2013;95-A:1600-1604

Funding Statement

- None declared

Author Contribution

J.W.A.M. den Teuling: Conception and design of the work, Drafting the manuscript, Approved the final manuscript.

B. S. Pauwels: Design of the work, Data collection, Data analysis, Drafting the initial manuscript, Approved the final manuscript.

L. Janssen: Conception and design of the work, Data analysis and critical revision of the manuscript, Approved the final manuscript.

- C. E. Wyers: Data collection and critical revision of the manuscript, Approved the final manuscript.

H. M. J.Janzing: Critical revision of the manuscript, Approved the final manuscript.

H. M. J.Janzing: Critical revision of the manuscript, Approved the final manuscript.
J. P.W. van den Bergh, Professor: Critical revision of the manuscript, Approved the final manuscript.

J. W. Morrenhof: Conception of the work, Critical revision of the manuscript, Approved the final manuscript.

Conflicts of Interest Statement

None declared

(c) 2017 Teuling et al. This is an open-access article distributed under the terms of the Creative Commons Attributions licence (CC-BY-NC), which permits unrestricted use, distribution, and reproduction in any medium, but not for commercial gain, provided the original author and source are credited. 